Supporting Information

\title{
A Polymer Electrolyte Membrane with High Ionic Conductivity and Enhanced Interfacial Stability for Lithium Metal Battery
}

Fengquan Liu, Fengjuan Bin, Jinxin Xue, Lu Wang, Yujie Yang, Hong Huo, Jianjun

Zhou ${ }^{*}$ and Lin $\mathrm{Li}^{*}$

Beijing Key Laboratory of Energy Conversion and Storage Materials, College of Chemistry, Beijing Normal University, Beijing, China 100875

${ }^{*}$ Correspondence and requests for materials should be addressed to L. Li. (lilinll@,bnu.edu.cn) or J. J. Zhou (E-mail:pla_zjj@,bnu.edu.cn). 


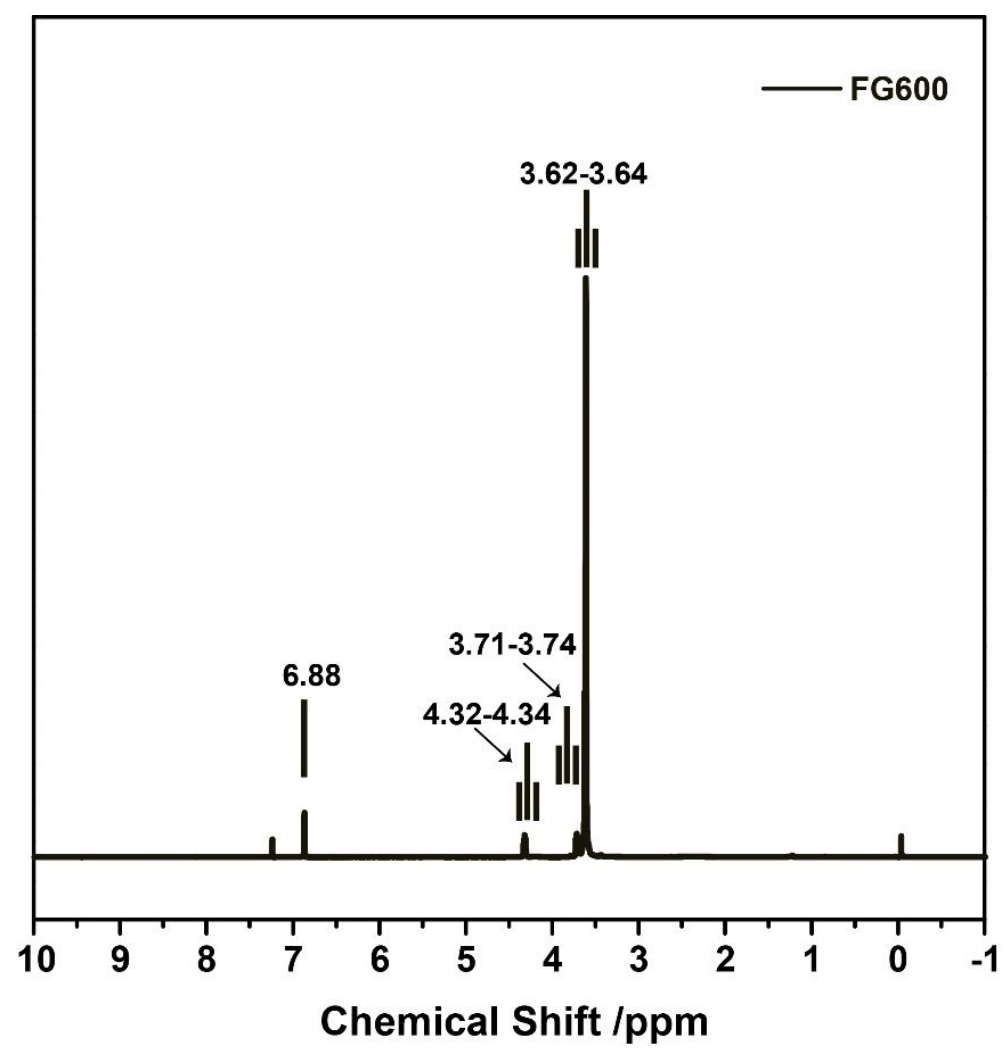

Figure S1. The ${ }^{1} \mathrm{H}$ NMR spectra of FG600. 

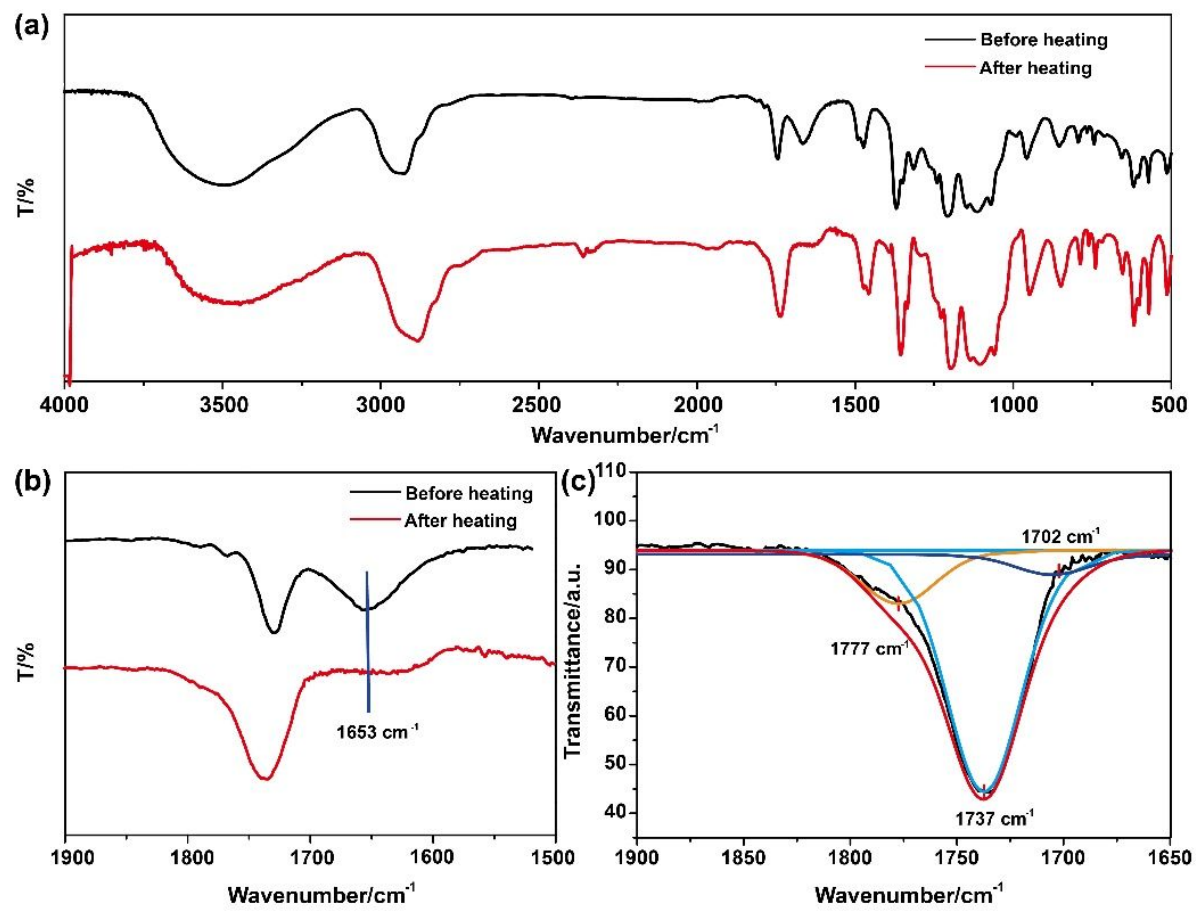

Figure S2. The FTIR spectra of the PEM before and after polymerization. 

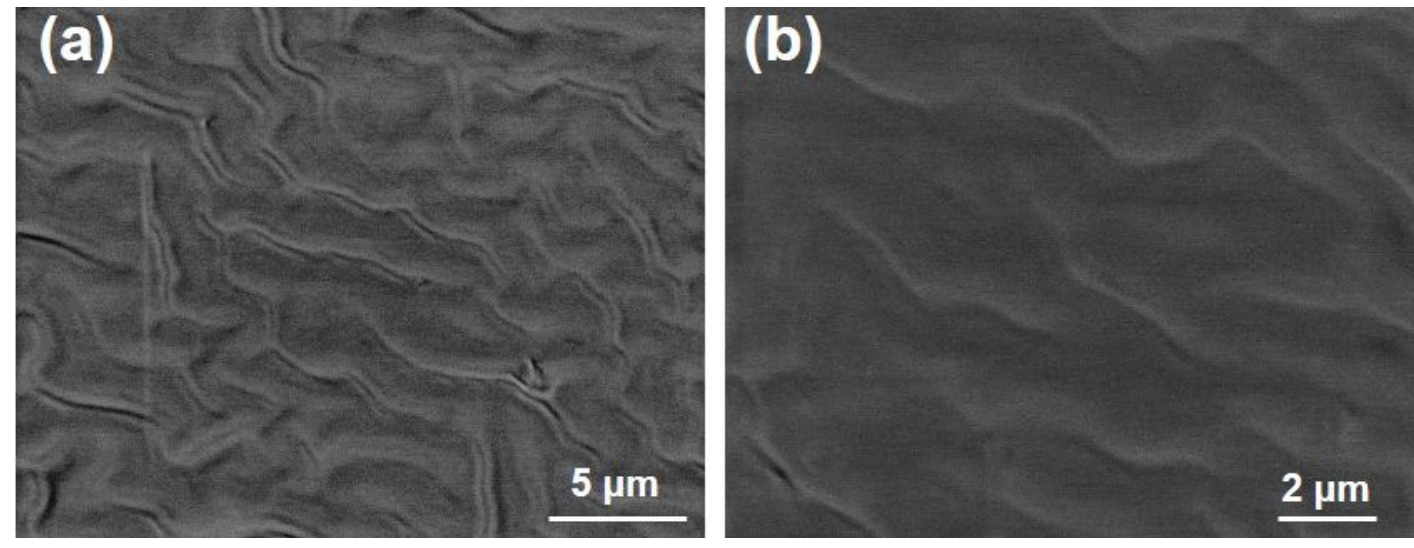

Figure S3. The surface SEM images of PEM. 


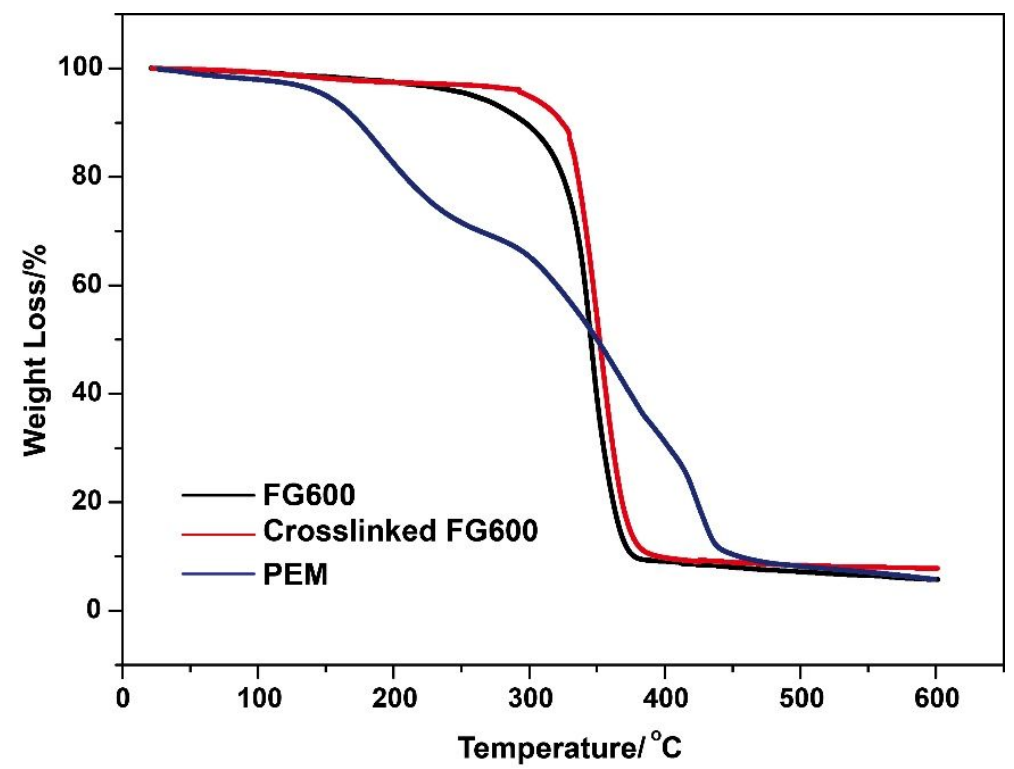

Figure S4. The TGA thermograms of FG600, crosslinked FG600 and PEM at the heating rate of $10^{\circ} \mathrm{C} \mathrm{min}^{-1}$ at $\mathrm{N}_{2}$ atmosphere. 


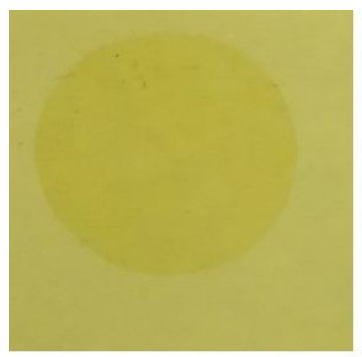

Flat

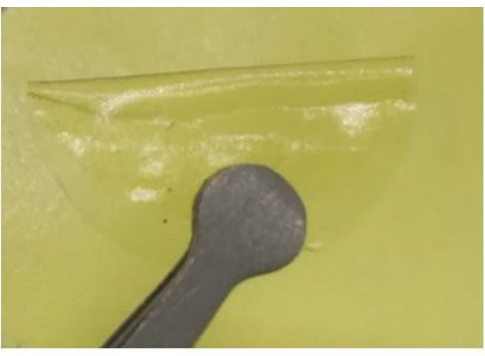

Bend

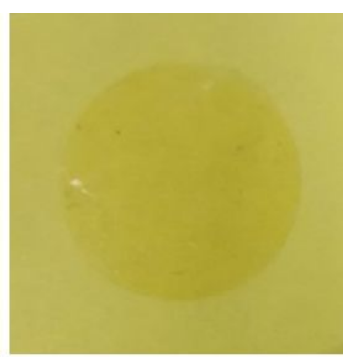

Recover

Figure S5. The optical photographs of PEM on the release paper at $110^{\circ} \mathrm{C}$. 


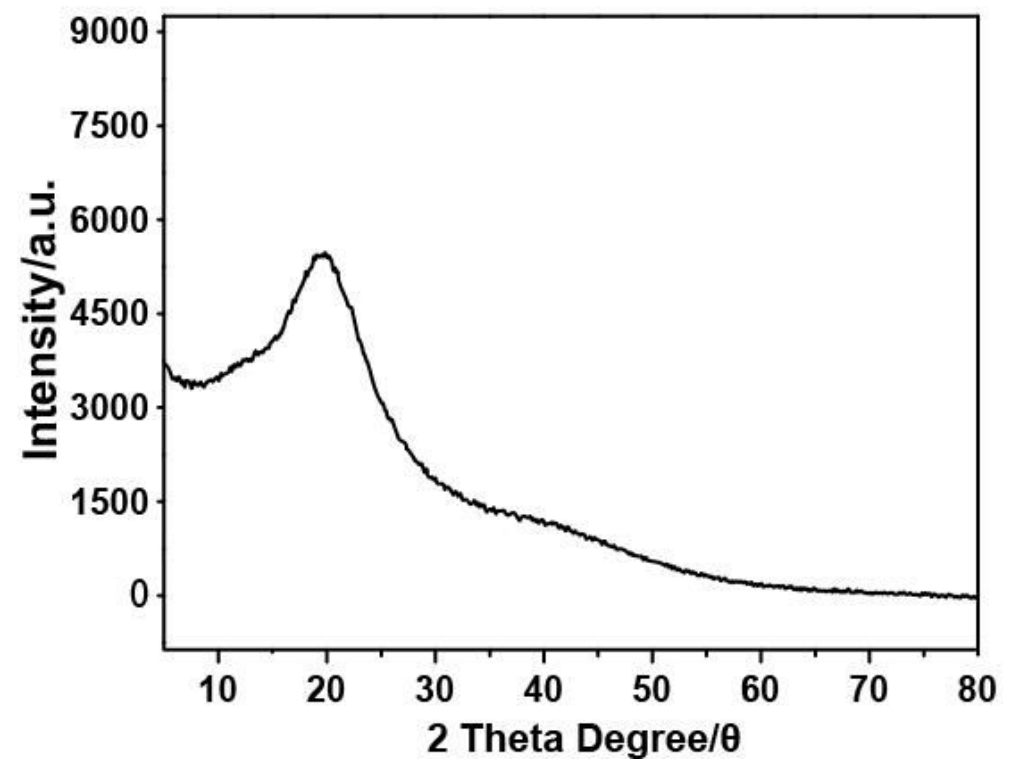

Figure S6. The XRD profile of PEM. 


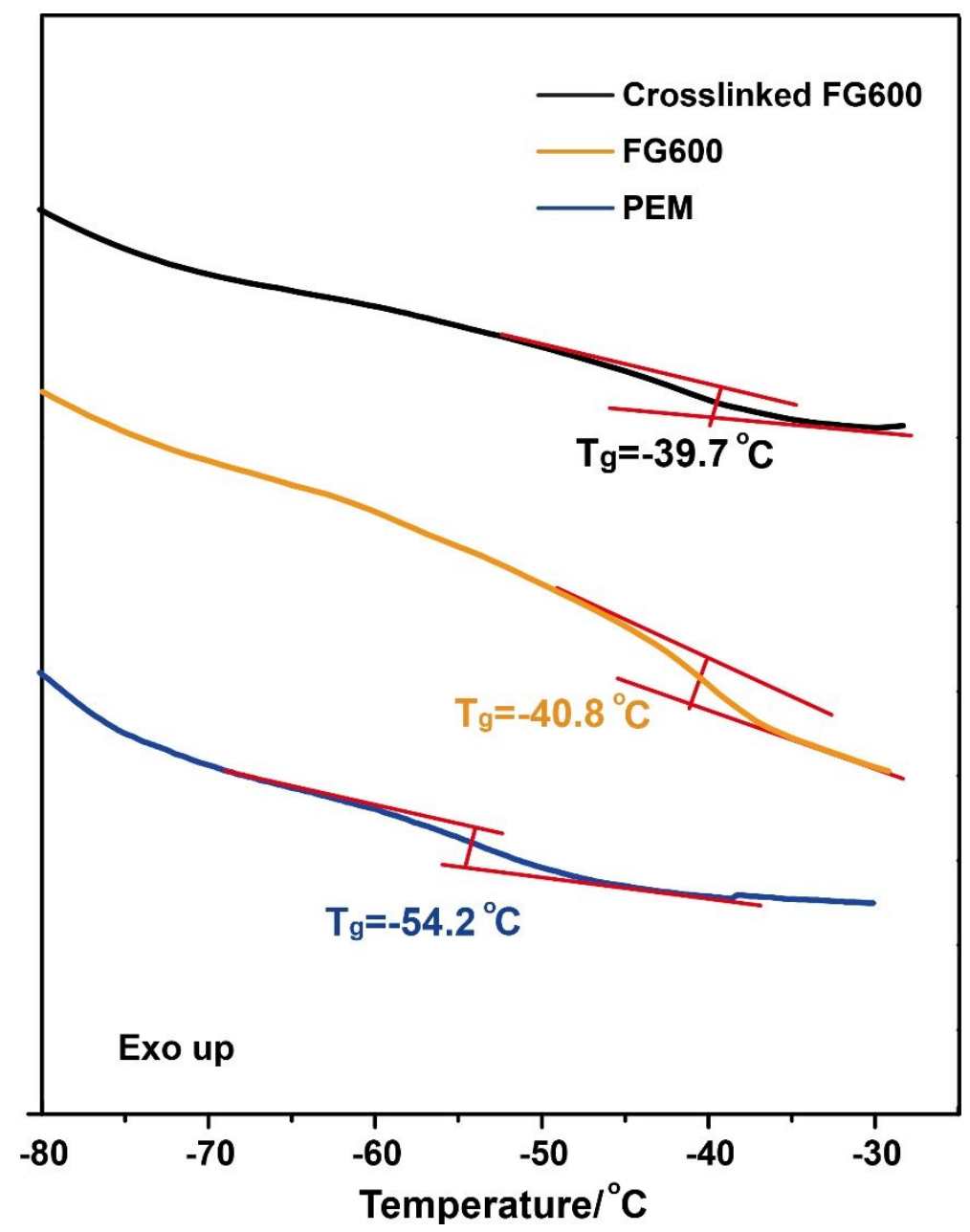

Figure S7. $T_{g}$ curves of FG600, crosslinked FG600 and PEM by DSC. 


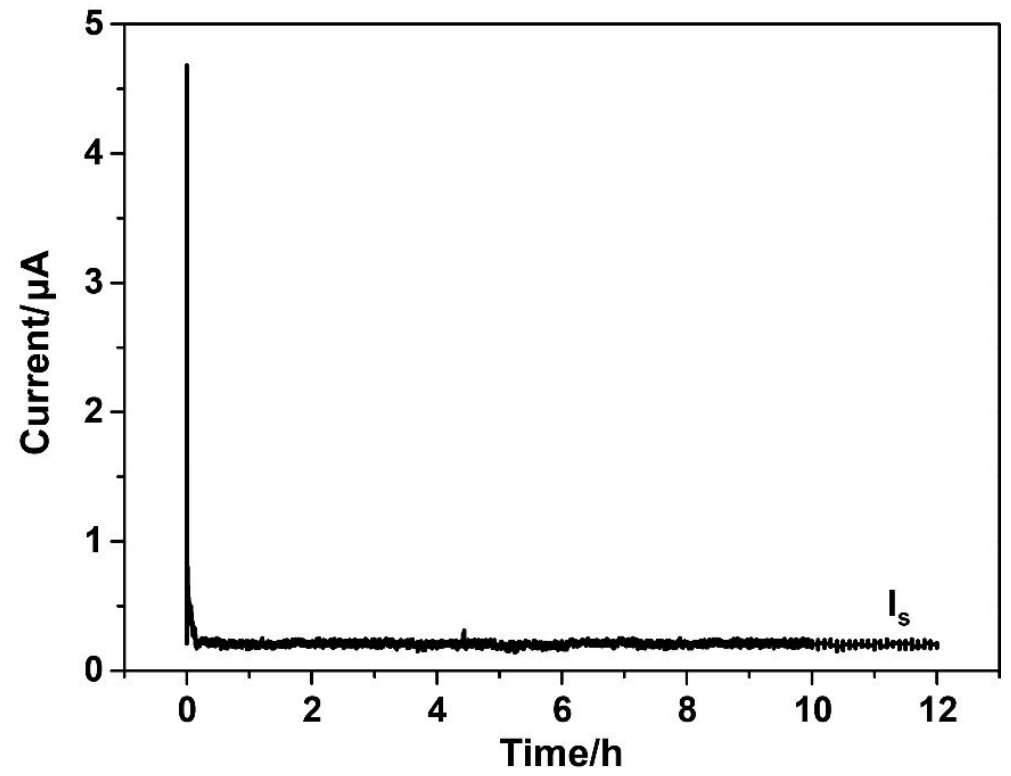

Figure S8. Potentiostatic coulometry measurement for PEM between two stainless steel electrodes at room temperature. 


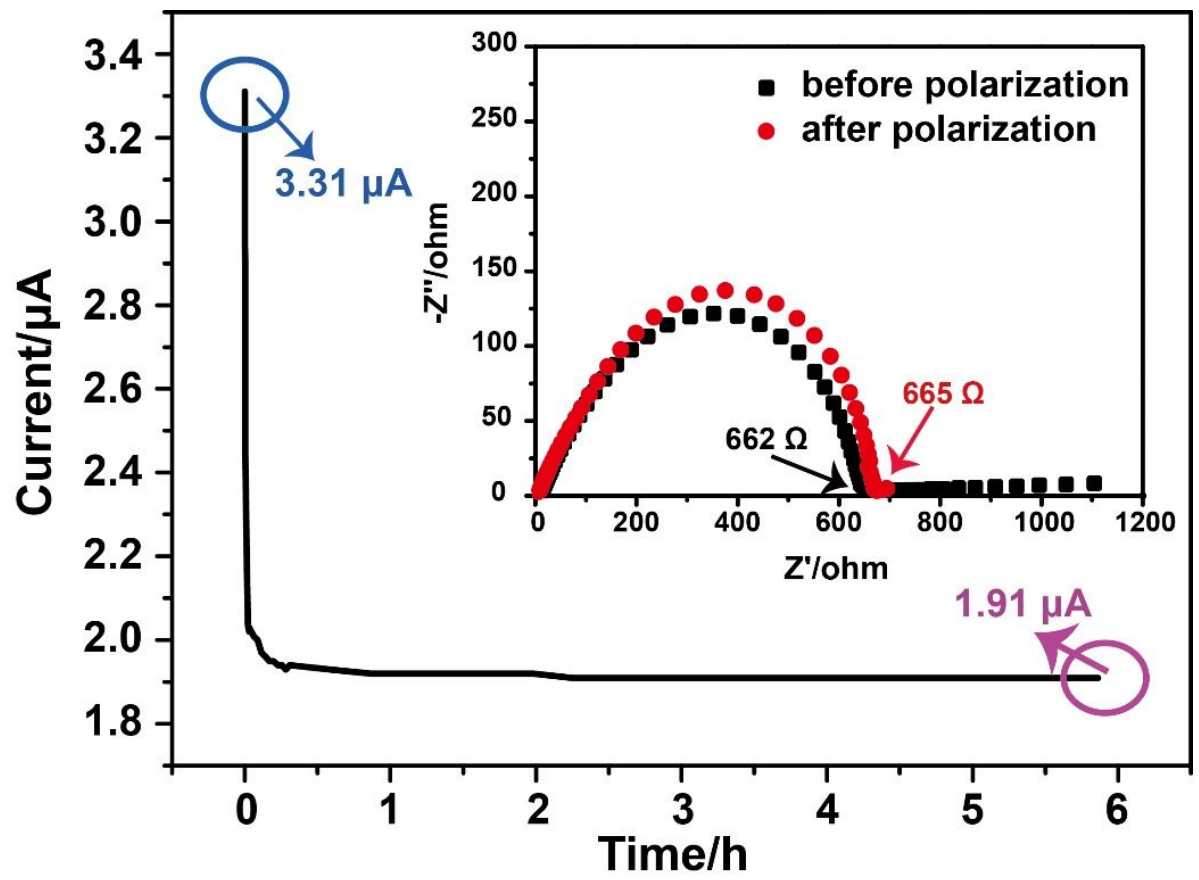

Figure S9. DC polarization curve and EIS of PEM before and after polarization for determining the $\mathrm{Li}^{+}$transference number. 


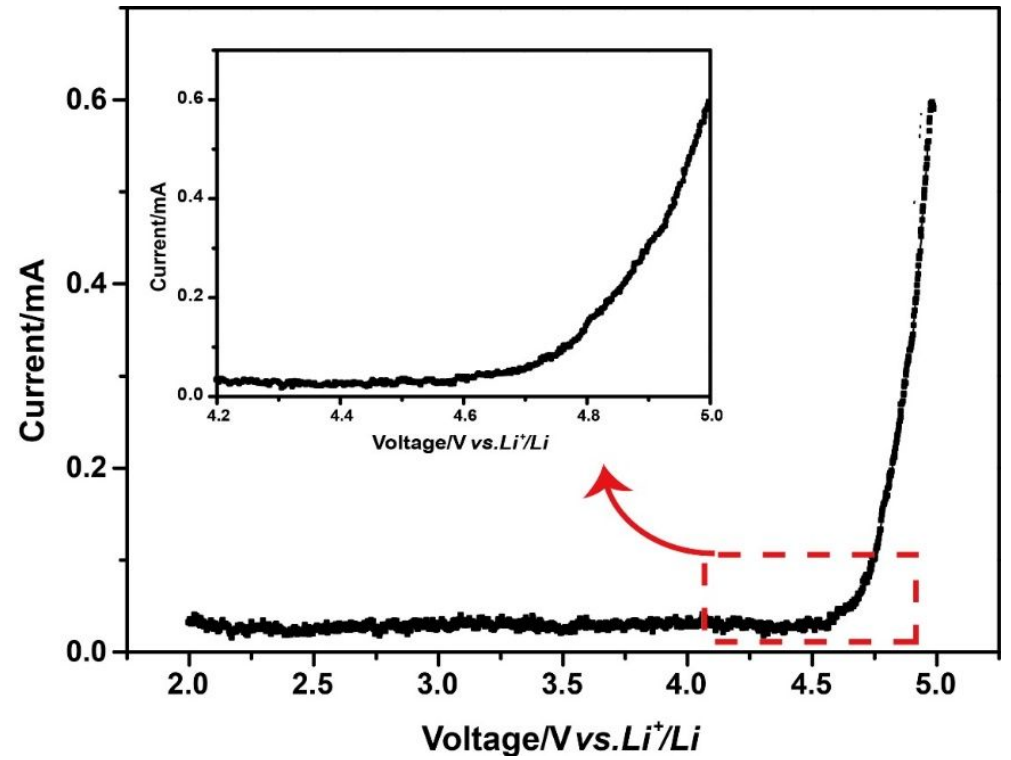

Figure S10. The linear sweep voltammetry curve of the PEM in the range of 2-5 V. 


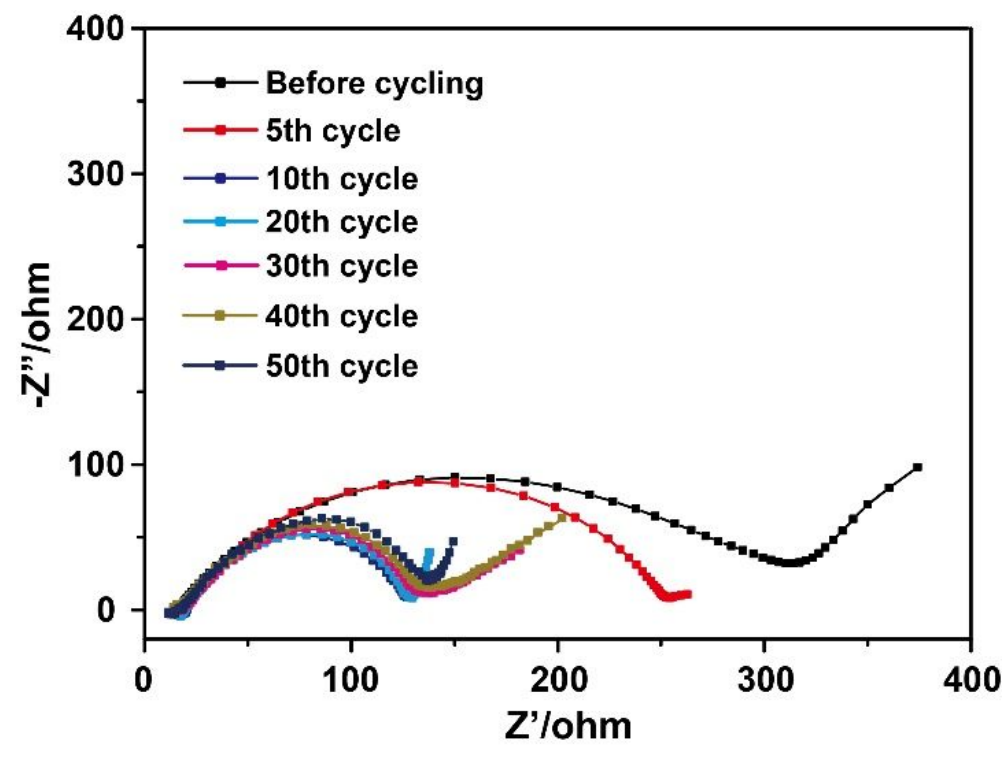

Figure S11. The EIS of $\mathrm{LiFePO}_{4}|\mathrm{PEM}| \mathrm{Li}$ cells at $40^{\circ} \mathrm{C}$. 


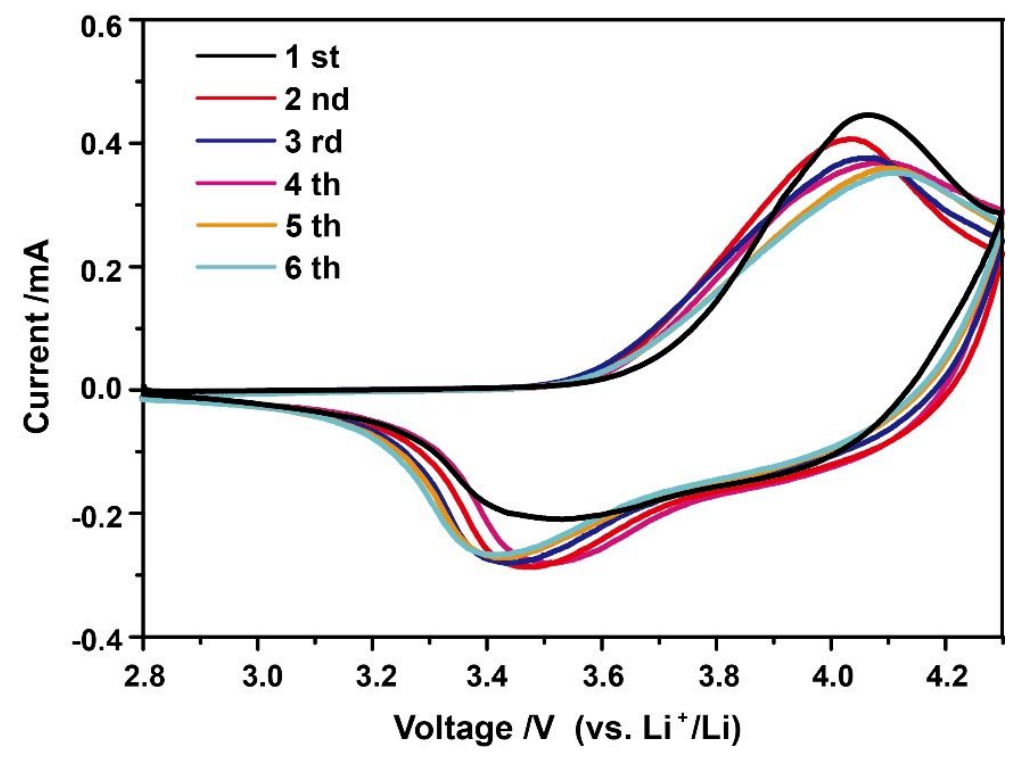

Figure S 12. CV profiles of NCM532|PEM|Li cells at a voltage range of $2.8-4.3 \mathrm{~V}$ at $40{ }^{\circ} \mathrm{C}$. 
Table S1 Band assignments for FTIR spectra of the PEM before and after polymerization.

\begin{tabular}{cc}
\hline Assignment & Band position, $\mathbf{c m}^{-1}$ \\
\hline$-\mathrm{OH}$ stretch & 3489 \\
$-\mathrm{CH}_{2}$ strech & 2851 \\
$-\mathrm{C}=\mathrm{O}$ carbonyl stretch & $1700 \sim 1800$ \\
$-\mathrm{C}=\mathrm{C}-$ strech & 1653 \\
$-\mathrm{CH} \mathrm{H}_{2}$ bend & 1452 \\
$-\mathrm{C}-\mathrm{O}-$ stretch & \\
\hline
\end{tabular}

The band at $3489 \mathrm{~cm}^{-1}$ comes from the stretching vibration of unreacted hydroxyl-terminated. The bands at $2851 \mathrm{~cm}^{-1}$ and $1452 \mathrm{~cm}^{-1}$ originate from the stretching and bending vibration of $-\mathrm{CH}_{2}$ in $\mathrm{EO}$ repeat unit. The broad band between 1700 and $1800 \mathrm{~cm}^{-1}$ can be assigned to the stretching vibration of $-\mathrm{C}=\mathrm{O}$ in ester group. The bands at $1194 \mathrm{~cm}^{-1}, 1130 \mathrm{~cm}^{-1}$ and $1093 \mathrm{~cm}^{-1}$ come from the stretching vibration of -C-O- in EO repeat unit. 
Table S2 Thermal stability among PEM and other polymer electrolytes.

\begin{tabular}{ccc}
\hline Polymer electrolytes & $\begin{array}{c}\text { Thermal decomposition } \\
\text { temperature }\left({ }^{\circ} \mathbf{C}\right)\end{array}$ & References \\
\hline PVA-CN/SN & 160 & {$[\mathrm{~S} 1]$} \\
PCE & 150 & {$[\mathrm{~S} 2]$} \\
MPEs & 290 & {$[\mathrm{~S} 3]$} \\
PPS-CPE & 200 & {$[\mathrm{~S} 4]$} \\
BMPE & 300 & {$[\mathrm{~S} 5]$} \\
P(BMA-AN-St) & 343 & {$[\mathrm{~S} 6]$} \\
Crosslinked FG600 & 300 & This work \\
\hline
\end{tabular}


Table S3 The glass transition temperatures of different polymer electrolytes.

\begin{tabular}{ccc}
\hline Polymer electrolytes & $\boldsymbol{T}_{\boldsymbol{g}}\left({ }^{\circ} \mathbf{C}\right)$ & References \\
\hline C-PEGDME & -49.2 & {$[\mathrm{~S} 7]$} \\
PEC-derived & -13 & {$[\mathrm{~S} 8]$} \\
PTMC-derived & -6 & {$[\mathrm{~S} 8]$} \\
$\left({ }^{76} \mathrm{PEOX}_{0.60}\right)\left({ }^{24} \mathrm{PE}_{0.40}\right)$ & -50 & {$[\mathrm{~S} 9]$} \\
PEO $_{34}-\mathrm{PC}$ & -55 & {$[\mathrm{~S} 10]$} \\
$\mathrm{CGPE}$ & -54 & {$[\mathrm{~S} 11]$} \\
CCPL & -28.8 & {$[\mathrm{~S} 12]$} \\
PTL-3 & -44 & {$[\mathrm{~S} 13]$} \\
PEM & -54.2 & This work \\
\hline
\end{tabular}


Table $\mathbf{S 4}$ The $\mathrm{Li}^{+}$ion transference numbers of polymer electrolytes.

\begin{tabular}{ccc}
\hline Polymer electrolytes & $\begin{array}{c}\mathbf{L i}^{+} \text {ion transference } \\
\text { number }\end{array}$ & References \\
\hline PAMM-based system & 0.51 & {$[\mathrm{~S} 14]$} \\
PETEA-based system & 0.47 & {$[\mathrm{~S} 15]$} \\
LATP/PAN-[PEO 8 -LiTFSI] & 0.32 & {$[\mathrm{~S} 16]$} \\
$\mathrm{CMC}^{2}$ & 0.46 & {$[\mathrm{~S} 17]$} \\
xPTHF ${ }_{10} \mathrm{SPE}$ & 0.53 & {$[\mathrm{~S} 18]$} \\
ipn-PEA & 0.65 & {$[\mathrm{~S} 19]$} \\
PEM & 0.58 & This work \\
\hline
\end{tabular}


Table S5 The cycling performances of $\mathrm{LiFePO}_{4}-\mathrm{Li}$ batteries with different electrolytes.

\begin{tabular}{cccc}
\hline Electrolyte component & $\begin{array}{c}\text { Working } \\
\text { condition }\end{array}$ & Capacity retention & References \\
\hline PEO+PEGDA+LiTFSI+DVB & $0.1 \mathrm{C}, 70^{\circ} \mathrm{C}$ & $105.7 \%, 20$ cycles & {$[\mathrm{S} 20]$} \\
BPT+Allyl PEO+Allyl & $0.1 \mathrm{C}, 60^{\circ} \mathrm{C}$ & $88 \%, 50$ cycles & {$[\mathrm{S} 21]$} \\
GA+DMPA+LiTFSI & & & \\
PEO+LLZO+LiTFSI & $0.2 \mathrm{C}, 55^{\circ} \mathrm{C}$ & $98.7 \%, 100$ cycles & {$[\mathrm{S} 22]$} \\
CPMEA+LLZO+CPMEA & $0.2 \mathrm{C}, 65^{\circ} \mathrm{C}$ & $\sim 100 \%, 100$ cycles & {$[\mathrm{S} 23]$} \\
PEO+WPU+LiTFSI & $1 \mathrm{C}, 60^{\circ} \mathrm{C}$ & $96 \%, 100$ cycles & {$[\mathrm{S} 24]$} \\
PEG+FG+LiTFSI & $0.1 \mathrm{C}, 25^{\circ} \mathrm{C}$ & $98.9 \%, 250$ cycles & This work \\
& & &
\end{tabular}

\section{Reference section}

(S1) Zhou, D.; He, Y. B.; Liu, R. L.; Liu, M.; Du, H. D.; Li, B. H.; Cai, Q.; Yang, Q. H.; Kang, F. Y., In Situ Synthesis of a Hierarchical All-Solid-State Electrolyte Based on Nitrile Materials for High-Performance Lithium-Ion Batteries. Adv. Energy Mater. 2015, 5, 1500353.

(S2) Ha, H. J.; Kil, E. H.; Kwon, Y. H.; Kim, J. Y.; Lee, C. K.; Lee, S. Y., UV-Curable Semi-Interpenetrating Polymer Network-integrated, Highly Bendable 
Plastic Crystal Composite Electrolytes for Shape-Conformable All-Solid-State Lithium Ion Batteries. Energy Environ. Sci. 2012, 5, 6491-6499.

(S3) Shim, J.; Bae, K. Y.; Kim, H. J.; Lee, J. H.; Kim, D. G.; Yoon, W. Y.; Lee, J. C., Solid Polymer Electrolytes Based on Functionalized Tannic Acids from Natural Resources for All-Solid-State Lithium-Ion Batteries. ChemSusChem 2015, 8, 4133-4138. Li, Y. J.; Fan, C. Y.; Zhang, J. P.; Wu, X. L., A Promising PMHS/PEO Blend Polymer Electrolyte for All-Solid-State Lithium Ion Batteries. Dalton Trans. 2018, 47, 14932-14937.

(S5) Gerbaldi, C.; Nair, J. R.; Meligrana, G.; Bongiovanni, R.; Bodoardo, S.; Penazzi, N., UV-Curable Siloxane-Acrylate Gel-Copolymer Electrolytes for Lithium-Based Battery Applications. Electrochim. Acta 2010, 55, 1460-1467.

(S6) Huang, W. Y.; Liao, Y. H.; Li, G. J.; He, Z. Y.; Luo, X. Y.; Li, W. S., $\begin{array}{llll}\text { Investigation } & \text { on } & \text { Polyethylene } & \text { Supported }\end{array}$ methacrylate-acrylonitrile-dtyrene) Terpolymer Based Gel Electrolyte Reinforced by Doping Nano-SiO 2 for High Voltage Lithium Ion Battery. Electrochim. Acta 2017, $251,145-154$

(S7) Cui, Y.; Liang, X.; Chai, J.; Cui, Z.; Wang, Q.; He, W.; Liu, X.; Liu, Z.; Cui, G.; Feng, J., High Performance Solid Polymer Electrolytes for Rechargeable Batteries: A Self-Catalyzed Strategy toward Facile Synthesis. Adv. Sci. 2017, 4, 1700174. 
Tominaga, Y., Ion-Conductive and Thermal Properties of a Synergistic Poly(ethylene carbonate)/Poly(trimethylene carbonate) Blend Electrolyte. Macromol. Rapid Commun. 2018, 39, 1800146.

(S9) Khurana, R.; Schaefer, J. L. S.; Archer, L. A.; Coates, G. W., Suppression of Lithium Dendrite Growth Using Cross-Linked Polyethylene/Poly(ethylene oxide) Electrolytes: A New Approach for Practical Lithium-Metal Polymer Batteries. J. Am. Chem. Soc. 2014, 136, 7395-7402.

(S10) Meabe, L.; Huynh, T. V.; Lago, N.; Sardon, H.; Li, C.; O'Dell, L. A.; Armand, M.; Forsyth, M.; Mecerreyes, D., Poly(ethylene oxide carbonates) Solid Polymer Electrolytes for Lithium Batteries. Electrochim. Acta 2018, 264, 367-375.

(S11) Natarajan, A.; Stephan, A. M.; Chan, C. H.; Kalarikkal, N.; Thomas, S., Electrochemical Studies on Composite Gel Polymer Electrolytes for Lithium Sulfur-Batteries. J. Appl. Polymer Sci. 2017, 134, 44594.

(S12) Zhang, J. J.; Yue, L. P.; Hu, P.; Liu, Z. H.; Qin, B. S.; Zhang, B.; Wang, Q. F.; Ding, G. L.; Zhang, C. J.; Zhou, X. H.; Yao, J. H.; Cui, G. L.; Chen, L. Q., Taichi-Inspired Rigid-Flexible Coupling Cellulose-Supported Solid Polymer Electrolyte for High-Performance Lithium Batteries. Sci. Rep. 2014, 4, 6272.

(S13) Porcarelli, L.; Gerbaldi, C.; Bella, F.; Nair, J. R., Super Soft All-Ethylene Oxide Polymer Electrolyte for Safe All-Solid Lithium Batteries. Sci. Rep. 2016, 6, 19892. 
(S14) Ma, Y.; Ma, J.; Chai, J.; Liu, Z.; Ding, G.; Xu, G.; Liu, H.; Chen, B.; Zhou, X.; Cui, G.; Chen, L., Two Players Make a Formidable Combination: In Situ Generated Poly(acrylic anhydride-2-methyl-acrylic acid-2-oxirane-ethyl ester-methyl methacrylate) Cross-Linking Gel Polymer Electrolyte toward $5 \mathrm{~V}$ High-Voltage Batteries. ACS Appl. Mater. Interfaces 2017, 9, 41462-41472.

(S15) Liu, M.; Zhou, D.; He, Y. B.; Fu, Y.; Qin, X.; Miao, C.; Du, H.; Li, B.; Yang, Q.-H.; Lin, Z.; Zhao, T. S.; Kang, F., Novel Gel Polymer Electrolyte for High-Performance Lithium-Sulfur Batteries. Nano Energy 2016, 22, 278-289.

(S16) Li, D.; Chen, L.; Wang, T. S.; Fan, L. Z., 3D Fiber-Network-Reinforced Bicontinuous Composite Solid Electrolyte for Dendrite-Free Lithium Metal Batteries. ACS Appl. Mater. Interfaces 2018, 10, 7069-7078.

(S17) Zhu, Y. S.; Xiao, S. Y.; Li, M. X.; Chang, Z.; Wang, F. X.; Gao, J.; Wu, Y. P., Natural Macromolecule Based Carboxymethyl Cellulose as a Gel Polymer Electrolyte with Adjustable Porosity for Lithium Ion Batteries. J. Power Sources $\mathbf{2 0 1 5}, 288,368-375$.

(S18) Mackanic, D. G.; Michaels, W.; Lee, M.; Feng, D. W.; Lopez, J.; Qin, J.; Cui, Y.; Bao, Z. N., Crosslinked Poly(tetrahydrofuran) as a Loosely Coordinating Polymer Electrolyte. Adv. Energy Mater. 2018, 8, 1800703.

(S19) Zeng, X. X.; Yin, Y. X.; Li, N. W.; Du, W. C.; Guo, Y. G.; Wan, L. J., Reshaping Lithium Plating/Stripping Behavior via Bifunctional Polymer Electrolyte for Room-Temperature Solid Li Metal Batteries. J. Am. Chem. Soc.2016, 138, $15825-15828$. 
(S20) Ben Youcef, H.; Garcia-Calvo, O.; Lago, N.; Devaraj, S.; Armand, M., Cross-Linked Solid Polymer Electrolyte for All-Solid-State Rechargeable Lithium Batteries. Electrochim. Acta 2016, 220, 587-594.

(S21) Shim, J.; Kim, L.; Kim, H. J.; Jeong, D.; Lee, J. H.; Lee, J. C., All-Solid-State Lithium Metal Battery with Solid Polymer Electrolytes Based on Polysiloxane Crosslinked by Modified Natural Gallic Acid. Polymer 2017, 122, $222-231$.

(S22) Chen, L.; Li, Y. T.; Li, S. P.; Fan, L. Z.; Nan, C. W.; Goodenough, J. B., PEO/Garnet Composite Electrolytes for Solid-State Lithium Batteries: From "Ceramic-in-Polymer" to "Polymer-in-Ceramic". Nano Energy 2018, 46, 176-184.

(S23) Zhou, W.; Wang, S.; Li, Y.; Xin, S.; Manthiram, A.; Goodenough, J. B., Plating a Dendrite-Free Lithium Anode with a Polymer/Ceramic/Polymer Sandwich Electrolyte. J. Am. Chem. Soc. 2016, 138, 9385-9388.

(S24) Bao, J.; Qu, X.; Qi, G.; Huang, Q.; Wu, S.; Tao, C.; Gao, M.; Chen, C., Solid Electrolyte Based on Waterborne Polyurethane and Poly(ethylene oxide) Blend Polymer for All-Solid-State Lithium Ion Batteries. Solid State Ionics 2018, 320, $55-63$. 